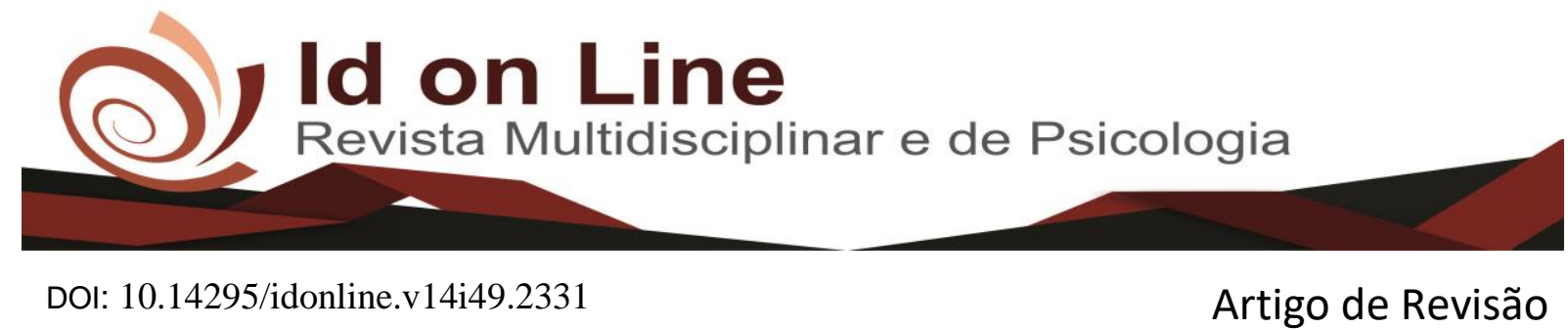

\title{
O uso da Música nos Cuidados Paliativos
}

\author{
Kevellyn Cruz Aguilera ${ }^{1}$; Isabelle Lima Mendes ${ }^{2}$; Modesto Leite Rolim Neto ${ }^{3}$
}

\begin{abstract}
Resumo: Os cuidados paliativos, associados à musicoterapia, pretendem aliviar e confortar os pacientes em final de vida. A terminalidade da vida configura-se como um período de adaptação e ressignificação de muitos conceitos prévios. Sendo assim, é necessário que haja um acompanhamento multidisciplinar desse enfermo enfocando nas suas necessidades apresentadas que podem ser físicas, espirituais ou psicossociais, oferecendo todo o suporte para a melhoria da qualidade de vida. Esse trabalho tem como objetivo apontar o que é realizado dentro da musicoterapia nos cuidados paliativos e se é efetivo o uso para a terminalidade da vida. Para isso, foi realizada uma revisão sistemática utilizando a base de dados e o Portal de Periódicos CAPES. Incluíram-se, nesta busca, os artigos publicados entre 2010 a 2019 , empregando os descritores MeSH: "music therapy" e "palliative care" com o operador boleano "AND”. Dentre os 431 artigos, 14 preencheram os critérios de elegibilidade. A maioria discursou sobre o bem-estar e alívio da dor com, principalmente, a prática receptiva da musicoterapia, tendo resultados positivos. O uso da música, como parte integrativa da terapia multidisciplinar dos cuidados paliativos, evidencia uma forma de conexão importante com a essência pessoal, ao passo que a pessoa que recorre à música tanto com o ato de cantar, como também escrever, tocar ou apenas ouvir, recorda, muitas vezes, memórias pregressas, reconectando o vínculo familiar e individual. Além disso, a musicoterapia ainda se alia ao estado de criatividade e arte, quando é avaliada a perspectiva de escrever suas próprias letras de músicas, e à capacidade de relaxamento e lazer, ao tocar instrumentos musicais ou escutar as músicas já gravadas, principalmente quando se correlaciona com sua cultura e sua preferência musical. Demonstrada a importância desse tema, por fim, é relevante que sejam feitos mais estudos relativos a essa temática com um maior rigor metodológico.
\end{abstract}

Palavras-chave: Fim de vida. Musicoterapia. Cuidados paliativos.

\section{The Use of Music in Palliative Care}

\begin{abstract}
Palliative care, associated with music therapy, is intended to relieve and comfort end-of-life patients. The terminality of life is configured as a period of adaptation and resignification of many previous concepts. Thus, there is a need for multidisciplinary monitoring of this patient focusing on their needs that may be physical, spiritual or psychosocial, offering all the support for improving the quality of life. This article aims to point out what is done within music therapy in palliative care and if it is effective to use for terminal life. For this, a systematic review was performed using the database and the CAPES Journal Portal. This search included articles published from 2010 to 2019 , using the descriptors MeSH: "music therapy" and "palliative care" with the Boolean operator "AND". Of the 431 articles, 14 met the eligibility criteria. Most spoke about well-being and pain relief with, mainly, the receptive practice of music therapy, having positive results. The use of music, as an integral part of multidisciplinary palliative care therapy, highlights an important form of connection with personal essence, whereas the person who uses music through singing as well as writing, playing or just listening often recalls past memories, reconnecting the family and individual bond. In addition, music therapy is still allied to the state of creativity and art, when evaluating the prospect of writing their own lyrics, and the ability to relax and play when playing musical instruments or listening to the songs already recorded, especially when playing. correlates with your culture and your musical preference. Demonstrating the importance of this theme, finally, it is relevant that further studies on this subject be made with greater methodological rigor.
\end{abstract}

Keywords: End-of-life; Music therapy. Palliative care.

\footnotetext{
${ }^{1}$ Graduação em Medicina. Faculdade de medicina Estácio - Juazeiro do Norte - CE. kevellynaguilera3@ gmail.com;

${ }^{2}$ Residência médica pela Faculdade de Medicina de Juazeiro do Norte. Professora de Propedêutica Médica da Faculdade de Medicina Estácio de Juazeiro do Norte , Brasil;

${ }^{3}$ Doutorado em Ciências da Saúde pela Universidade Federal do Rio Grande do Norte. Professor PhD em Saúde Pública USP. modestorolim@yahoo.com.br.
} 


\section{Introdução}

A "terminalidade" envolve múltiplas situações clínicas. Na atualidade está passando por uma transição conceitual dados os avanços provocados pelos cuidados paliativos. Observase que a terminologia, "paciente em final de vida", não provoca um fim em si, mas uma situação onde se pode provocar o mapeamento de coisas inacabadas. Desse modo, a musicoterapia adentra como ferramenta técnica de alívio e equilíbrio da dor e do sofrimento psíquico.

Estima-se que cerca de 40 milhões de pessoas no mundo precisem de cuidados paliativos, número que se ampliou com o processo de envelhecimento populacional e consequente incremento na proporção das doenças crônicas, porém apenas 14\% recebem tratamento (OMS, 2018).

A musicoterapia é entendida como um processo terapêutico dinâmico que visa reduzir a percepção de dor e sofrimento, além de controlar o humor, melhorando o senso de controle e relaxamento, sendo conduzida por um musicoterapeuta que facilita a promoção da saúde do cliente por meio de experiências musicais e desenvolvimento de relações pessoais através do dinamismo proposto pelo uso da música que se constitui uma forma propícia de aprimoramento cognitivo, facilitando o desenvolvimento de uma comunicação mais eficaz. (WARTH et al., 2019; SCHIMID et al., 2018; WARTH et al., 2016).

A musicoterapia é muito utilizada na terminalidade da vida como forma de auxílio à expressão dos sentimentos particulares associados a experiências pregressas psicossociais e espirituais presentes na vivência do paciente e/ou dos seus familiares, propiciando uma mais coerente comunicação e atendimento de instâncias espirituais muitas vezes desconhecidas pelo próprio paciente, além de contribuir com o alívio de sintomas físicos, o que levou a uma maior empregabilidade dos musicoterapeutas nos hospitais que possuem o setor de cuidados paliativos. (WARTH et al., 2019; BRADT; DILEO, 2011).

O uso da música como forma de terapia se deve aos efeitos que são provocados no corpo humano, podendo ser citados uma melhora dos níveis pressóricos e de frequência respiratória e cardíaca, além de relaxamento muscular, melhora do sono, diminuição da ansiedade e uma melhora da fadiga, se popularizando mais após a canadense musicoterapeuta, Susan Munro, realizar uma descrição sistemática do uso da musicoterapia em vários domínios pessoais, tais como, físico e psicológico (WARTH et al., 2019; SCHIMID et al., 2018; SCHIMID; OSTERMANN, 2010). 
O sintoma álgico influi de diversos meios negativamente no processo de adoecimento, pois contribui para uma modulação bioquímica, através do estresse emocional evocado, que aumenta os níveis de catecolaminas e cortisol, facilitando no caso do câncer, por exemplo, a uma progressão tumoral, além disso, há uma piora da qualidade de vida, considerando um aumento dos níveis de tristeza, ansiedade e humor deprimido. Tratando-se de uma experiência complexa e peculiar a cada pessoa, a dor é influenciada pelos aspectos biopsicossociais, dependendo, portanto, da forma com que o indivíduo está emocionalmente. (FERNANDO et al., 2019; WARTH et al., 2019; SCHIMID et al., 2018).

Considerando o exposto acima, surge a necessidade de haver um manejo mais eficaz da dor, através ou das modalidades farmacológicas ou das não farmacológicas. O uso de medicações analgésicas consegue modular o sintoma álgico, sendo escalonadas de acordo com a necessidade, porém muitas vezes levam a vários efeitos colaterais, à tolerância da medicação, não tendo mais o efeito esperado pela dose feita, além da dependência, principalmente a opioides. Nesse contexto, quando não há manejo adequado dos sintomas com a terapia convencional, inclusive como iniciativa dos familiares e do paciente, tornam-se importante as iniciativas não-farmacológicas complementares, sendo a musicoterapia bastante empregada com essa finalidade de melhorar a qualidade de vida, através da redução desses sintomas estressantes. (FERNANDO et al., 2019; WARTH et al., 2019; ZENG et al., 2018).

Na musicoterapia há o aspecto de indução das emoções e sua modulação positiva, impactando diretamente no humor, que gera a distração do sofrimento físico e emocional, que vincula a terapia às reminiscências felizes e que ajuda a recobrar, nesta conjuntura, sua alegria e reduzir o negativismo frente à sua condição. Foi demonstrada, em geral, uma diminuição dos níveis tensionais e uma melhora do bem-estar do paciente, porém é necessário avaliar que, de acordo com o musicoterapeuta e com os diferentes métodos de musicoterapia, existe promoção de respostas distintas e mesmo que a maioria dos pacientes possua uma percepção positiva da musicoterapia e a alie em seu arsenal terapêutico, não é a totalidade, precisando numa etapa anterior à aplicação, o paciente ser questionado e optar sobre o uso ou não dessa ferramenta. (RAMIREZ et al.,2018; PORTER et al., 2017; WARTH et al., 2015).

Nessa perspectiva de cuidado holístico, o uso da musicoterapia, para a diminuição dos níveis de ansiedade e estresse, mostra-se como uma terapêutica auxiliar, que associada aos outros tratamentos, possui um bom custo-benefício, necessitando de mais estudos que confirmem as vantagens dessa modalidade nos cuidados paliativos objetivando a ampliação e melhoria da qualidade de vida, principalmente considerando a faixa etária dos adultos e idosos. 
Desta forma, surge a pergunta norteadora de pesquisa, baseada no acrônimo PICO, onde: P: Pacientes em cuidados paliativos; I: Musicoterapia; CO: Adultos e idosos em final de vida. Desse modo, delineou-se a pergunta norteadora: "Quais as atuações e a praticabilidade da musicoterapia em pacientes adultos e idosos em fim de vida nos cuidados paliativos?".

O objetivo geral do presente estudo foi descrever as evidências que apontam as atuações e a praticabilidade da musicoterapia nos pacientes adultos e idosos em cuidados paliativos. Para isso foi necessário também: a) Filtrar as evidências que abordam o uso da musicoterapia nos cuidados paliativos em pacientes adultos e idosos em final de vida; $b$ ) Averiguar, através dos estudos elegíveis, a praticabilidade clínica da musicoterapia nos cuidados paliativos e, c) Analisar as atuações realizadas pela musicoterapia em pacientes adultos e idosos em final de vida.

\section{Método}

Para obter os objetivos da pesquisa e melhor elucidar as questões, foi optado por empregar uma Revisão Sistemática da literatura. A pesquisa foi realizada de ordem prática, visto que possui o propósito de como realizar de forma mais eficiente a musicoterapia nos cuidados paliativos ofertada para adultos e idosos e o tipo de pesquisa bibliográfica escolhida foi a Revisão Sistemática, porque fornece melhor as evidências reais, provendo uma ideia do custo-benefício do que é analisado, havendo a avaliação crítica de várias pesquisas simultaneamente, abrangendo assim uma maior contingente de pessoas envolvidas na pesquisa, em contraponto com a Revisão Tradicional que não segue um rigor metodológico podendo chegar a vários fins incluídos na mesma pesquisa (KAURA, 2015; GIL, 2017).

A revisão sistemática trata-se, portanto, de um estudo retrospectivo e secundário, encontrando os resultados discordantes e os similares, tentando eliminar os vieses. Possui um rigor na redação da avaliação ao passo que os artigos selecionados são avaliados de acordo com sua relevância e validade, dependendo assim da qualidade das informações contidas nesses artigos, tendo um caráter imparcial na combinação e interpretação dos resultados (KAURA, 2015; SAMPAIO; MANCINI, 2007; GUANILO; TAKAHASHI, 2011).

A pesquisa foi feita no período de 27 de dezembro de 2019 a 28 de dezembro de 2019 para obtenção dos dados. Executou-se o levantamento bibliográfico nas seguintes bases de dados: Biblioteca Virtual de Saúde (BVS) e US National Library of Medicine National 
Institutes of Health (PubMed). Para a formatação do trabalho, utilizar-se-á o padrão definido nas normas da ABNT.

Foram realizados os seguintes passos para a construção da Revisão Sistemática, a partir do protocolo Prisma. Disponível em: http://prisma-statement.org/.

Primeira Etapa: Seleção de Hipóteses ou Questões para Investigação: Após realizar leitura breve sobre o tema, o questionamento levantado foi sobre os benefícios advindos da musicoterapia associados aos cuidados paliativos. Logo, a questão norteadora da pesquisa foi: "Quais as intervenções e a efetividade da musicoterapia em pacientes adultos e idosos nos cuidados paliativos?”.

Segunda Etapa: Amostragem ou Busca na Literatura: Nesse segundo momento, iniciou-se a seleção nas seguintes bases de dados, Biblioteca Virtual de Saúde (BVS) e US National Library of Medicine National Institutes of Health (PubMed). Para tanto, foram usados os descritores: "palliative care" e "music therapy".

Critérios de inclusão

- Disponibilidade do texto completo nas bases de dados e/ou Potal de Periódico CAPES;

- Pesquisas feitas em adultos e idosos em final de vida;

- Ter sido publicado nos últimos 10 anos na Pubmed e na BVS;

- Estudos escritos em português ou inglês;

- Os estudos que abordavam custo-benefício no uso da musicoterapia e melhoria na qualidade de vida em ciudados paliaitivos.

Critérios de exclusão

- Estudos que envolvam crianças e adolescentes;

- Abordagem centrada na visão familiar;

- Artigos repetidos nas duas bases de dados;

- Os que envolviam outras abordagens dos cuidados paliativos que não a musicoterapia;

- Estudos que, para possuir acesso, precisava de assinatura ou pagamento.

Terceira Etapa: Categorização dos estudos: Na pesquisa utilizando-se a combinação dos descritores MeSH: "palliative care" e "music therapy" com o operador boleano "AND" foram encontrados 431 resultados nas bases de dados propostas. Ao aplicar os filtros e posterior elegibilidade obteve-se um (n): 96 artigos na BVS. E na PubMed, com os filtros e posterior elegibilidade, obteve-se um (n): 25 artigos. Após aplicar os critérios de exclusão restou n: 14 artigos. 
Quarta etapa: Avaliação dos Estudos Incluídos na Revisão (Análise de Dados): Representa a análise dos resultados vistos nos artigos inclusos na amostra. Sendo assim, após a análise foram discutidos três pontos principais, sendo eles: as categorias de musicoterapia usadas na prática, o emprego da musicoterapia no cuidado paliativo e os principais benefícios advindos da musicoterapia no cuidado paliativo.

Quinta etapa: Interpretação dos resultados: Os artigos lidos foram comparados e extraídos as informações de acordo com os critérios de elegibilidade para compor a discussão deles.

Sexta etapa: Síntese do Conhecimento ou Apresentação da Revisão: Corresponde ao término da redação do texto, havendo o reconhecimento das principais evidências e resultados encontrados a partir dos artigos escolhidos.

\section{Resultados}

A princípio, foram encontrados 431 artigos nas bases de dados e no Portal de Periódicos - CAPES. Em seguida, ao ser aplicado os critérios de exclusão e filtros, mantiveramse 14 artigos selecionados, sendo utilizados após leitura integral. A figura 1 representa o protocolo seguida para a escolha, adaptado do protocolo PRISMA.

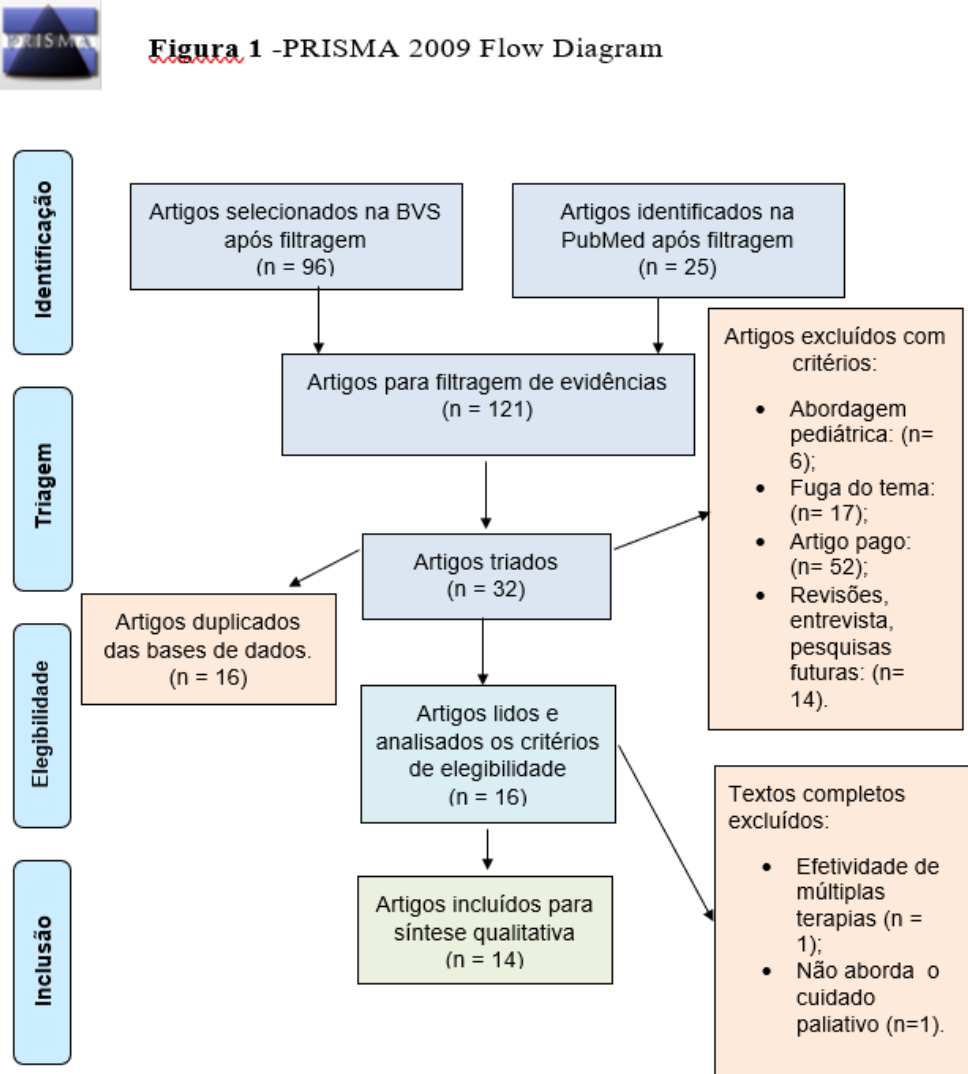

Fonte: Adaptado do Protocolo Prisma. Disponível em: http://prismastatement.org/. 
Após a seleção dos artigos que comporiam a síntese quantitativa para o presente artigo, houve a uma triangulação teórica para posterior formação da Tabela 1. Foi relatado em apenas um artigo a não comprovação estatisticamente significativa de nenhum parâmetro biopsicossocial (WARTH et al., 2015).

Tabela 1 - Resultados encontrados com o (n) final de artigos selecionados

\begin{tabular}{|c|c|c|c|c|c|}
\hline Ano & Título & Revista & Autores & Amostra & Principal achado \\
\hline 2019 & $\begin{array}{l}\text { "Adjunctive Effects of a } \\
\text { Short Session of Music } \\
\text { on Pain, Low-mood and } \\
\text { Anxiety Modulation } \\
\text { among Cancer Patients" } \\
\text { - A Randomized } \\
\text { Crossover Clinical Trial }\end{array}$ & $\begin{array}{l}\text { Indian Journal } \\
\text { of Palliative } \\
\text { Care - IJPC }\end{array}$ & $\begin{array}{l}\text { Fernando, } \\
\text { et al., }\end{array}$ & $\begin{array}{l}\text { O estudo foi realizado } \\
\text { com adultos em uma } \\
\text { unidade de cuidados } \\
\text { terciários de pacientes } \\
\text { com câncer, sendo } \\
\text { utilizado um total de } 24 \\
\text { pacientes. }\end{array}$ & $\begin{array}{l}\text { Houve uma melhora } \\
\text { estatisticamente significativa } \\
\text { dos níveis de ansiedade, dor e } \\
\text { humor deprimido com a } \\
\text { musicoterapia. }\end{array}$ \\
\hline 2018 & $\begin{array}{l}\text { A randomised } \\
\text { controlled pilot and } \\
\text { feasibility study of } \\
\text { music therapy for } \\
\text { improving the quality of } \\
\text { life of hospice } \\
\text { inpatients }\end{array}$ & $\begin{array}{l}\text { BMC } \\
\text { Palliative } \\
\text { Care }\end{array}$ & $\begin{array}{l}\text { Porter, et } \\
\text { al., }\end{array}$ & $\begin{array}{l}\text { O estudo incluiu } 52 \\
\text { pacientes que foram } \\
\text { avaliados por } 1 \text { ano, } \\
\text { sendo } 25 \text { no grupo de } \\
\text { intervenção e } 26 \text { no } \\
\text { grupo de controle. }\end{array}$ & $\begin{array}{l}\text { Visualizou-se uma melhora } \\
\text { substancial do bem-estar } \\
\text { existencial, porém o autor } \\
\text { recomenda que esse estudo seja } \\
\text { refeito com um número maior } \\
\text { de pacientes, para se ter valores } \\
\text { estatisticamente significativos. }\end{array}$ \\
\hline 2018 & $\begin{array}{l}\text { EEG-Based Analysis of } \\
\text { the Emotional Effect of } \\
\text { Music Therapy on } \\
\text { Palliative Care Cancer } \\
\text { Patients }\end{array}$ & $\begin{array}{l}\text { Frontiers in } \\
\text { Psychology }\end{array}$ & $\begin{array}{l}\text { Ramirez, } \\
\text { et al., }\end{array}$ & $\begin{array}{l}\text { Foi conduzido o estudo } \\
\text { com } 50 \text { pacientes em } \\
\text { cuidados paliativos, } \\
\text { sendo escolhido } \\
\text { aleatoriamente } 20 \text { para o } \\
\text { grupo de intervenção e } \\
20 \text { para o grupo controle. }\end{array}$ & $\begin{array}{l}\text { Ao se analisar o traçado do } \\
\text { eletroencefalograma, notou-se } \\
\text { uma mudança significativa dos } \\
\text { parâmetros entre o início e o } \\
\text { final da sessão o que indica um } \\
\text { efeito positivo na questão } \\
\text { emocional. }\end{array}$ \\
\hline 2017 & $\begin{array}{l}\text { A critical realist } \\
\text { evaluation of a music } \\
\text { therapy intervention in } \\
\text { palliative care }\end{array}$ & $\begin{array}{l}\text { BMC } \\
\text { Palliative } \\
\text { Care }\end{array}$ & $\begin{array}{l}\text { Porter, et } \\
\text { al., }\end{array}$ & $\begin{array}{l}\text { Foi recrutado um total } \\
\text { de } 16 \text { pacientes em um } \\
\text { hospice na Irlanda do } \\
\text { Norte entre Junho de } \\
2016 \text { e fevereiro de } \\
2017 .\end{array}$ & $\begin{array}{l}\text { Foi relatado pelos participantes } \\
\text { uma melhoria de aspectos } \\
\text { emocionais, físicos, } \\
\text { psicológicos, espirituais e } \\
\text { sociais, como melhor } \\
\text { comunicação com a família. }\end{array}$ \\
\hline 2016 & $\begin{array}{l}\text { Trajectories of } \\
\text { Terminally Ill Patients' } \\
\text { CardiovascularRespons } \\
\text { e to Receptive Music } \\
\text { Therapy in Palliative } \\
\text { Care }\end{array}$ & $\begin{array}{l}\text { Journal of } \\
\text { Pain and } \\
\text { Symptom } \\
\text { Management. }\end{array}$ & $\begin{array}{l}\text { Warth, et } \\
\text { al., }\end{array}$ & $\begin{array}{l}84 \text { pacientes } \\
\text { participaram do estudo, } \\
\text { sendo divididos em } 2 \\
\text { grupos, sendo um com o } \\
\text { musicoterapeuta com } \\
\text { sessões de relaxamento e } \\
\text { um com prática baseadas } \\
\text { em "mindfulness" e } \\
\text { relaxamento para } \\
\text { avaliação do sistema } \\
\text { nervoso autônomo. }\end{array}$ & $\begin{array}{l}\text { Houve uma redução do tônus } \\
\text { vascular simpático, traduzindo } \\
\text { um relaxamento dos níveis de } \\
\text { estresse e dor no grupo que } \\
\text { participou das sessões de } \\
\text { musicoterapia. }\end{array}$ \\
\hline 2016 & $\begin{array}{l}\text { Favored subjects and } \\
\text { psychosoial needs in } \\
\text { music therapy in } \\
\text { terminally ill cancer } \\
\text { patients: a content } \\
\text { analysis }\end{array}$ & $\begin{array}{l}\text { BMC } \\
\text { Palliative } \\
\text { Care }\end{array}$ & $\begin{array}{l}\text { Preissler, } \\
\text { et al., }\end{array}$ & $\begin{array}{l}41 \text { pacientes em } \\
\text { cuidados paliativos, } \\
\text { admitidos entre Junho } \\
2012 \text { e outubro de } 2014 \text {, } \\
\text { em um hospital na } \\
\text { Alemanha, realizaram } \\
\text { acompanhamento com } 2 \\
\text { musicoterapeutas, } \\
\text { perfazendo } 166 \text { sessões. }\end{array}$ & $\begin{array}{l}\text { Foram obtidas } 7 \text { categorias } \\
\text { pessoais e nove dimensões de } \\
\text { necessidades psicossociais que } \\
\text { eram mais retratadas nas } \\
\text { sessões, demonstrando que a } \\
\text { musicoterapia auxilia na } \\
\text { abordagem dessas necessidades } \\
\text { psicossociais e espirituais. }\end{array}$ \\
\hline 2016 & $\begin{array}{l}\text { Effect of Music } \\
\text { Therapy on Pain and } \\
\text { Anxiety Levels of }\end{array}$ & $\begin{array}{l}\text { Indian Journal } \\
\text { Palliative } \\
\text { Care - IJPC }\end{array}$ & $\begin{array}{l}\text { Krishnas } \\
\text { wamy, } \\
\text { Nair }\end{array}$ & $\begin{array}{l}\text { Tratou-se de um estudo } \\
\text { quantitativo que utilizou } \\
14 \text { pacientes de um } \\
\text { serviço de atendimento } \\
\text { médico terciário que }\end{array}$ & $\begin{array}{l}\text { Houve uma melhora } \\
\text { estatisticamente significativa } \\
\text { dos níveis de nocicepção. }\end{array}$ \\
\hline
\end{tabular}




\begin{tabular}{|c|c|c|c|c|c|}
\hline & $\begin{array}{l}\text { Cancer Patients: A Pilot } \\
\text { Study }\end{array}$ & & & $\begin{array}{l}\text { tinham relato de dor de } \\
\text { moderada a severa. }\end{array}$ & \\
\hline 2015 & $\begin{array}{l}\text { Music Therapy in } \\
\text { Palliative Care }\end{array}$ & $\begin{array}{l}\text { Deutsches } \\
\text { Ärzteblatt } \\
\text { International }\end{array}$ & $\begin{array}{l}\text { Warth, et } \\
\text { al., }\end{array}$ & $\begin{array}{l}\text { Foi realizado o estudo } \\
\text { com } 84 \text { pacientes } \\
\text { institucionalizados em } \\
\text { suporte paliativo, sendo } \\
\text { divididos no grupo da } \\
\text { musicoterapia e em um } \\
\text { de controle com outras } \\
\text { técnicas de relaxamento. }\end{array}$ & $\begin{array}{l}\text { Demonstrou-se que a } \\
\text { musicoterapia era mais efetiva } \\
\text { do que os exercícios de } \\
\text { relaxamento em promover } \\
\text { bem-estar emocional e } \\
\text { tranquilidade. }\end{array}$ \\
\hline 2015 & $\begin{array}{l}\text { Treating Palliative Care } \\
\text { Patients with Pain with } \\
\text { the Body Tambura:A } \\
\text { Prospective Case Study } \\
\text { at St. Joseph's Hospice } \\
\text { for Dying Destitute in } \\
\text { Dindigul South India }\end{array}$ & $\begin{array}{l}\text { Indian Journal } \\
\text { Palliative } \\
\text { Care - IJPC }\end{array}$ & $\begin{array}{l}\text { Dietrich, } \\
\text { et al., }\end{array}$ & $\begin{array}{l}\text { Compôs o estudo um } \\
\text { total de } 10 \text { pacientes que } \\
\text { eram atendidos em um } \\
\text { hospice no Sul da Índia. } \\
\text { Todos os pacientes } \\
\text { estavam desassistidos de } \\
\text { cuidadores e/ou } \\
\text { familiares. }\end{array}$ & $\begin{array}{l}\text { Foi observado uma melhora } \\
\text { importante nos parâmetros da } \\
\text { dor física clinicamente, sendo } \\
\text { que a melhora foi gradualmente } \\
\text { aumentando de acordo com a } \\
\text { quantidade de sessões com o } \\
\text { musicoterapeuta. }\end{array}$ \\
\hline 2015 & $\begin{array}{l}\text { Effects of vibroacoustic } \\
\text { stimulation in music } \\
\text { therapy for palliative } \\
\text { care patients: a } \\
\text { feasibility study }\end{array}$ & $\begin{array}{l}\text { BMC } \\
\text { Complementa } \\
\text { ry and } \\
\text { Alternative } \\
\text { Medicine }\end{array}$ & $\begin{array}{l}\text { Warth, et } \\
\text { al., }\end{array}$ & $\begin{array}{l}\text { O estudo foi realizado } \\
\text { com } 9 \text { pacientes com } \\
\text { câncer em estágio } \\
\text { avançado que receberam } \\
\text { sessão de } 30 \text { minutos de } \\
\text { musicoterapia, sendo } \\
\text { aplicada uma escala } \\
\text { visual analógica para } \\
\text { obtenção dos resultados. }\end{array}$ & $\begin{array}{l}\text { Não foram encontradas } \\
\text { diferenças estatisticamente } \\
\text { significativas do pré e pós teste } \\
\text { realizado nos domínios do } \\
\text { bem-estar, dor e relaxamento. } \\
\text { Porém o autor afirma que são } \\
\text { necessários mais estudos. }\end{array}$ \\
\hline 2014 & $\begin{array}{l}\text { Significados e sentidos } \\
\text { da identidade musical } \\
\text { de pacientes e } \\
\text { familiares sob } \\
\text { cuidados paliativos } \\
\text { oncológicos*. }\end{array}$ & $\begin{array}{l}\text { Revista } \\
\text { eletrônica de } \\
\text { enfermagem. }\end{array}$ & $\begin{array}{l}\text { Silva, } \\
\text { Alvin, } \\
\text { Marcon }\end{array}$ & $\begin{array}{l}\text { Foram } 33 \text { usuários da } \\
\text { Rede Feminina de } \\
\text { Combate ao Câncer de } \\
\text { Maringá que } \\
\text { participaram dos } \\
\text { encontros musicais, mas } \\
\text { apenas } 12 \text { entraram para } \\
\text { os estudos (sete } \\
\text { pacientes e cinco } \\
\text { familiares), por terem } \\
\text { participado de } 3 \\
\text { encontros ou mais. }\end{array}$ & $\begin{array}{l}\text { Os autores chegaram à } \\
\text { conclusão de que, o uso da } \\
\text { musicoterapia no contexto dos } \\
\text { cuidados paliativos, associados } \\
\text { a escolhas musicais próprias do } \\
\text { grupo selecionado compunham } \\
\text { uma forma de conforto e } \\
\text { consequente maneira de lidar } \\
\text { melhor com todo seu entorno } \\
\text { social. }\end{array}$ \\
\hline 2014 & $\begin{array}{l}\text { Perceived outcomes of } \\
\text { music therapy with } \\
\text { Body Tambura in end } \\
\text { of life care }- \text { a } \\
\text { qualitativepilot study }\end{array}$ & $\begin{array}{l}\text { BMC } \\
\text { Palliative } \\
\text { Care }\end{array}$ & $\begin{array}{l}\text { Teut, et } \\
\text { al., }\end{array}$ & $\begin{array}{l}\text { Foi realizada uma } \\
\text { entrevista após o } \\
\text { tratamento com sessões } \\
\text { de musicoterapia com o } \\
\text { instrumento Body } \\
\text { Tambura, perfazendo um } \\
\text { total de } 11 \text { pessoas, } \\
\text { sendo } 8 \text { pacientes com } \\
\text { câncer em estágio } \\
\text { avançado e } 3 \text { familiares } \\
\text { em um hospital em } \\
\text { Berlim. }\end{array}$ & $\begin{array}{l}\text { Após a entrevista, foi visto que } \\
\text { as sessões de musicoterapia } \\
\text { com o Body Tambura eram } \\
\text { concebidas como um } \\
\text { instrumento de relaxamento, } \\
\text { associando-se a simbolismos } \\
\text { próprios, além de ser vista } \\
\text { como uma forma de fomentar o } \\
\text { vínculo familiar. }\end{array}$ \\
\hline 2013 & $\begin{array}{l}\text { Encontros musicais } \\
\text { como recurso em } \\
\text { cuidados paliativos } \\
\text { oncológicos a usuários } \\
\text { de casas de apoio*. }\end{array}$ & $\begin{array}{l}\text { Revista } \\
\text { Escola } \\
\text { Enfermagem } \\
\text { USP }\end{array}$ & $\begin{array}{l}\text { Silva, } \\
\text { Sales }\end{array}$ & $\begin{array}{l}20 \text { usuários da Rede } \\
\text { Feminina de Combate ao } \\
\text { Câncer de Maringá } \\
\text { participaram dos } \\
\text { encontros musicais, mas } \\
\text { apenas } 7 \text { deles, por } \\
\text { terem participado de } 3 \\
\text { ou mais encontros, } \\
\text { entraram no estudo } \\
\text { qualitativo e } \\
\text { fenomenológico. }\end{array}$ & $\begin{array}{l}\text { Os autores chegaram à } \\
\text { conclusão de que a } \\
\text { musicoterapia auxiliou os } \\
\text { participantes a terem um maior } \\
\text { entendimento e significado } \\
\text { quanto ao processo de morte e } \\
\text { o de existência e propósito. }\end{array}$ \\
\hline 2013 & $\begin{array}{l}\text { Music Therapy Reduces } \\
\text { Pain in Palliative } \\
\text { Care Patients: A } \\
\text { Randomized Controlled } \\
\text { Trial. }\end{array}$ & $\begin{array}{l}\text { Journal of } \\
\text { Pain and } \\
\text { Symptom } \\
\text { Management. }\end{array}$ & $\begin{array}{l}\text { Gutgsell, } \\
\text { et al., }\end{array}$ & $\begin{array}{l}\text { Foi realizada pesquisa } \\
\text { com } 200 \text { pacientes no } \\
\text { período de } 2009 \text { a } 2011, \\
\text { utilizando um grupo de } \\
\text { controle e um com } \\
\text { musicoterapia, sendo } \\
\text { realizado tanto música } \\
\text { ao vivo e técnicas de } \\
\text { relaxamento. }\end{array}$ & $\begin{array}{l}\text { A partir da escala de dor visual } \\
\text { analógica utilizada pela equipe } \\
\text { de enfermagem foi vista uma } \\
\text { redução estatisticamente } \\
\text { significativa dos níveis de dor } \\
\text { referidas pelos pacientes que } \\
\text { fizeram o método da } \\
\text { musicoterapia. }\end{array}$ \\
\hline
\end{tabular}

Fonte: Autoria própria. 


\section{Discussão}

A terminalidade da vida impõe para os cuidados paliativos uma série de desafios em que surgem questionamentos de ordem psicológica, social e espiritual, fazendo-se necessário o uso de técnicas que ajudem o enfermo a se expressar, atendendo-o de forma holística, melhorando sua qualidade de vida e bem-estar (WARTH et al., 2019; WARTH et al., 2016). Sendo assim, com o intuito de melhor correlacionar as ideias quanto à terminalidade da vida, é apresentada a Figura 2.

Figura 2 - Mapa Conceitual sobre terminalidade da vida

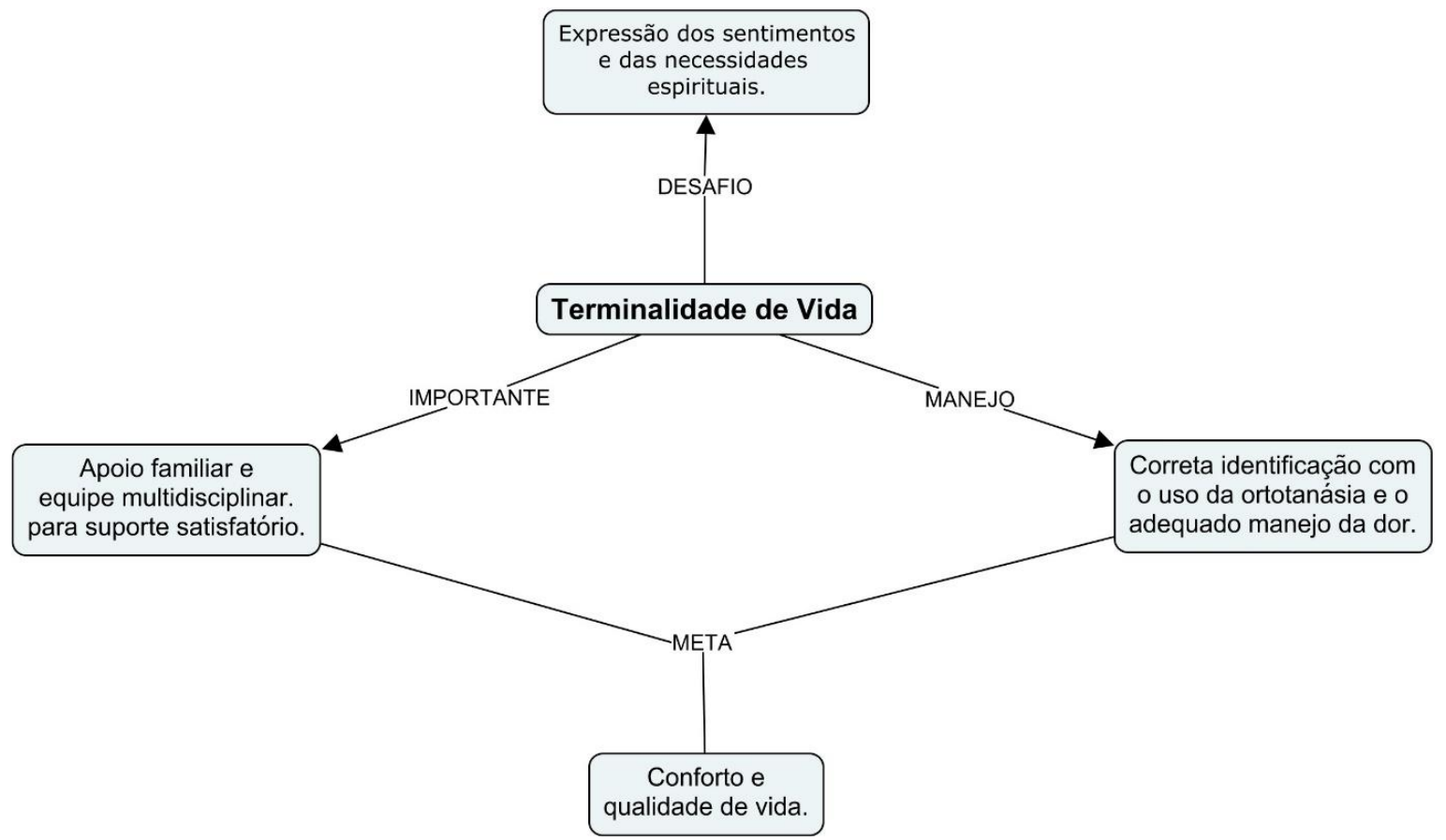

Fonte: Autoria própria.

A finitude da vida representa um período em que muitas fragilidades individuais são expostas, o que leva a uma necessidade de enfrentamento delas por parte da equipe que acompanha o enfermo, porém, muitas vezes, a equipe de saúde encara o final da vida como um período com pouco a ser feito pelo paciente. Nesse contexto, os cuidados paliativos elucidaram muitos questionamentos sobre o que pode ser realizado nesse período da vida, atendendo a demandas sociais, espirituais, psicológicas e físicas dessa população com doenças fora de possibilidade terapêutica curativa (CAIRES et al., 2014; SEKI; GALHEIGO, 2010). A OMS, 
em 2002, já incorporava esse conceito de conforto e alívio do sofrimento biopsicossocial, tanto aos pacientes quanto aos familiares, aos cuidados paliativos, ressaltando a importância de serem iniciados precocemente ao diagnóstico da patologia ameaçadora da vida.

Desse modo, para facilitar o suporte dado aos pacientes em fim de vida que fazem acompanhamento com os cuidados paliativos, há várias terapias complementares, sendo as mais comuns a massoterapia, terapia em grupo e a musicoterapia. Essa última, foi instituída, pois, ao passo que é desenvolvida, no processo terapêutico, uma relação entre o musicoterapeuta e o paciente, é alcançada um meio de minimizar o estresse, a ansiedade, aumentando assim a disposição para se realizar as atividades diárias. A musicoterapia se fundamenta, nesse contexto, no uso da música como fonte para imaginação, criatividade ou até mesmo como forma recreativa e de relaxamento (WARTH et al., 2019; PORTER et al., 2018; ZENG et al., 2018; WARTH et al., 2016; BRADT; DILEO, 2011; SCHIMID; OSTERMANN, 2010).

A música, que já vem sendo implantada como terapia desde o milênio passado, é um instrumento multidisciplinar tornando-se uma linguagem universal e multifacetada que abrange vários domínios tanto físicos, quanto emocionais e cognitivos, a partir do momento em que auxilia na comunicação, diminuindo a inquietação e aumentando o engajamento com suas práticas tanto ativas, em que o paciente canta, escreve uma música e/ou dança, toca instrumentos musicais, quanto passiva, em que ele escuta ou apenas conversa com o musicoterapeuta. Trata-se, portanto, de um elo criado entre o musicoterapeuta e o paciente, tendo a vantagem de ser facilmente implantada e possuir baixos custos. (FERNANDO et al., 2019; BUNN et al., 2018; SCHIMID et al., 2018).

Ademais, há uma aceitação da musicoterapia, tanto pelos médicos, quanto pela maioria dos pacientes, destacando a necessidade de conhecer o perfil do cliente para o início da terapia, como uma técnica que traz benefícios, sobretudo na melhoria da qualidade de vida. (PORTER et al., 2017; WARTH et al., 2015; KORCZAK et al., 2013).

É relevante que o profissional musicoterapeuta, antes de iniciar as sessões, predefina com o paciente, já que a flexibilidade desses quesitos foi demonstrada como positiva pelos estudos, o tempo de cada consulta que pode normalmente se estender por até duas a três horas por semana, a periodicidade entre elas, o local preferido e até mesmo o tipo de terapia - tendo uma maior eficácia ao serem empregadas músicas ao vivo ao invés das gravadas pelo maior dinamismo, inclusive mais proporcionado com a forma ativa da musicoterapia, ao engajar mais o cliente que usa sua voz ou instrumentos - e a escolha das músicas, porque os sons que foram considerados agradáveis pelo paciente aumentaram os níveis de dopamina e os que não foram, 
diminuíram esses níveis. Ademais, a religiosidade também apresenta influência na efetividade da musicoterapia, fortalecendo a fé e a esperança do enfermo e dos seus familiares (RAMIREZ et al., 2018; PORTER et al., 2017; PREISSLER et al., 2016; ARCHIE; BRUERA; COHEN, 2013).

A família e os cuidadores, inseridos nesse contexto, precisam ser também contemplados, considerando que são um dos pilares dos cuidados paliativos e envolvem-se em todo o processo de sofrimento do enfermo, tendo também benefícios ao usarem o artifício da musicoterapia com seus familiares, principalmente se a música for inserida na cultura dos mesmos, oferecendo uma forma de comunicação entre eles e aumento do vínculo familiar, podendo até os pacientes escreverem músicas dedicadas aos familiares, oferecendo, assim, uma recordação do ente querido. A musicoterapia, por aumentar o conforto e o bem-estar dos pacientes e familiares, muitas vezes, acaba sendo requerida quando há insucesso das medidas farmacológicas (WARTH, et al., 2019; SCHIMID et al., 2018; ZENG et al., 2018; MCCONNEL et al., 2016; SILVA; SALES, 2013).

A música, dentro de seus aspectos rítmicos e sonoros, quando já reconhecida e possuidora de significância pessoal, adquire um melhor engajamento do paciente com o musicoterapeuta, facilitando a expressão dos sentimentos e consequente melhoria da comunicação interpessoal, trazendo à tona reminiscências, levando assim, para um maior estado de bem-estar do paciente e da família que consegue retornar vínculos importantes com essas sessões (WARTH et al., 2019; CAIRES et al., 2014; GUTGSELL et al., 2013).

$\mathrm{Na}$ terminalidade da vida, a musicoterapia pode ser empregada em inúmeras enfermidades, tais como insuficiência cardíaca congestiva, câncer e doenças neurodegenerativas, sendo realizadas intervenções com o uso da música e sons, com práticas tanto ativas, quanto receptivas que objetivam envolver o paciente em um ambiente individualizado de acordo com suas necessidades no estágio que se encontra, seja para revigorar-se com músicas improvisadas ou mesmo para recordar momentos passados com músicas gravadas. (WARTH et al., 2019; PORTER et al., 2018; BRADT; DILEO, 2011; SCHIMID; OSTERMANN 2010). Sendo retratada na Figura 3 os principais tópicos pertinentes ao uso da musicoterapia. 
Figura 3 - Mapa conceitual sobre musicoterapia

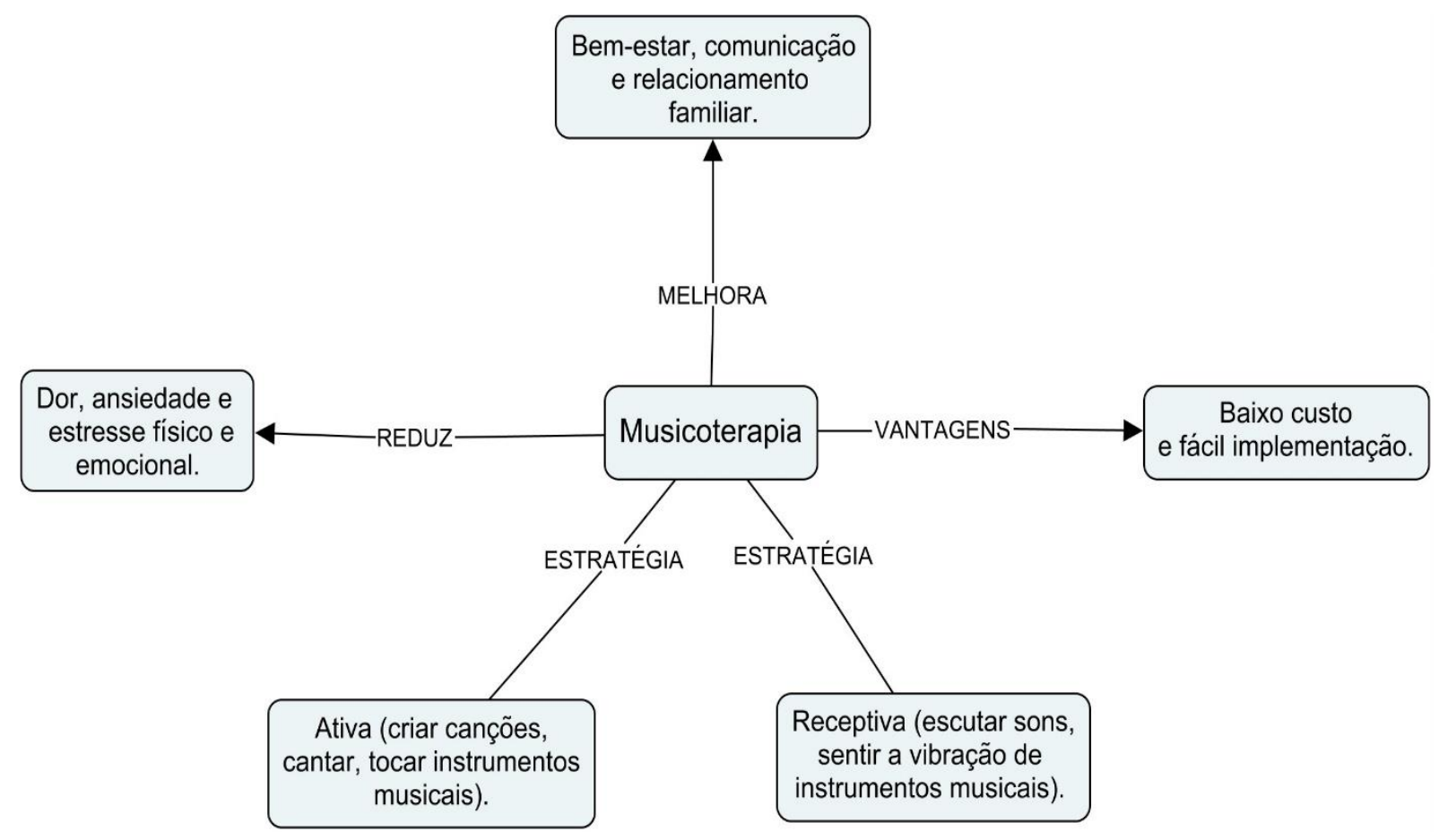

Fonte: Autoria própria

Quando se verifica as vantagens da musicoterapia em relação a outras formas de tratamento farmacológicas, confirma-se que é uma técnica com maior perfil de segurança e um mínimo de efeitos colaterais, diferenciando-se ainda da música medicinal, pois, ao passo que, essa se propõe a reproduzir músicas gravadas - previamente selecionadas por estudos administradas por profissionais da saúde que não precisam necessariamente dispor de treinamento por musicoterapeuta, a musicoterapia envolve, além das músicas gravadas, sons ao vivo ou de forma interativa de acordo com o perfil do paciente coordenado por um musicoterapeuta (TEUT et al., 2014; ARCHIE; BRUERA; COHEN, 2013).

A musicoterapia, tanto na forma ativa, quanto receptiva, apresenta-se, em associação aos cuidados paliativos, como uma configuração de atendimento holístico prestado a indivíduos com prognóstico ruim ou com doenças sem potencial de cura e é usualmente a escolha terapêutica adjuvante preferida nos 240ospice. Ela é definida em horários flexíveis ajustados às preferências do participante e preestabelecida suas metas de acordo com os aspectos psicossociais, sendo individualizada também pelo estado físico do enfermo e procurando incrementar a relação entre a equipe de saúde e o paciente (SCHMID et al., 2018; RAMIREZ et al., 2018; PORTER et al., 2017). 
Quando é realizada de forma passiva ou receptiva, pode ser acionada durante a música técnicas de estimulação vibroacústica e ativa ou recreativa em que se criam as próprias músicas - podendo, inclusive, existir um significado especial para os amigos e familiares - ou tocam instrumentos, dando a sensação de diversão e maior significância para si mesmo (PORTER et al., 2017; MCCONNEL et al., 2016; WARTH et al., 2015).

A forma receptiva, que foi a primeira a ser implementada em conjunto aos cuidados paliativos, é uma opção mais viável a depender do nível das limitações físicas do paciente, empregada ou com músicas previamente gravadas com significância para a pessoa que recebe o tratamento ou com sons produzidos pelos instrumentos musicais: gongos, didjeridu, pau-dechuva, tambor de oceano, monocórdio e Body Tambura. Esse último foi desenvolvido pelos musicoterapeutas alemães, primeiramente para pacientes em coma, com o intuito de produzir vibrações e energias para áreas específicas do corpo - dorso, pulmão, estômago e genitais sendo colocado ou próximo ou diretamente sobre o corpo do paciente. (PORTER et al., 2018; MCCONNEL et al., 2016; DIETRICH et al., 2015; TEUT et al., 2014).

O instrumento musical, Body Tambura, promove uma melhora de diversos parâmetros tanto subjetivos pelo conforto proporcionado, relaxamento profundo do corpo e da mente, mudanças positivas na autopercepção e crescimento do vínculo familiar, quanto objetivos pela diminuição da frequência cardíaca, pressão arterial e manutenção do nível da respiração, contribuindo para o aumento da qualidade de vida. Outro instrumento que é utilizado na terapêutica paliativa similar ao Body Tambura é o "singing chair", porém de acordo com os estudos, não houve mudança significativa após o uso desta técnica nos domínios da dor, relaxamento e bem-estar, cabendo ressaltar que nessa pesquisa não foi realizada à randomização tampouco o controle da amostra e foi incluída uma pequena parcela, não significativa da população estudada (DIETRICH et al., 2015; WARTH et al., 2015; TEUT et al., 2014).

Sintomas comuns em pacientes adultos e idosos nos cuidados paliativos como dor, ansiedade e oscilações do humor, que podem interferir diretamente na qualidade de vida do indivíduo, não conseguem o manejo satisfatório com a terapêutica farmacológica. E, ao passarem pela musicoterapia, houve uma redução da atividade alfa do lobo frontal esquerdo que pode melhorar o humor depressivo, além de uma mudança no seu estado emocional inicial, referindo menos fraqueza no final da consulta do que no início, ocorrendo de acordo com o aumento do significado da música para o indivíduo, um processo mais expressivo de autorreflexão, ao lidar com a situação de cuidados paliativos que se encontra, e, menor articulação dos problemas, considerando que ele irá relembrar dos momentos marcados por 
determinada música de forma mais vívida, o que o fará analisar todo o entorno da situação de forma mais saudosa, pautada nessas sensações relembradas (RAMIREZ et al., 2018; PREISSLER et al., 2016; ARCHIE; BRUERA; COHEN, 2013).

A modulação do sensório da nocicepção é verificada, principalmente quando realizada a musicoterapia em pacientes portadores de câncer, tendo um alívio da sensação álgica na escala de dor moderada a severa. Já na diminuição de ansiedade, um dos que provou a interação positiva foi o que a redimensionou como consequência do relaxamento (PORTER et al., 2017; KRISHNASWAMY; NAIR, 2016; WARTH et al., 2015).

Isso reverbera na parte emocional, tanto na forma de musicoterapia receptiva que possui um maior impacto na calmaria do paciente, quanto na forma ativa que foca mais em entender pela linguagem corporal os pensamentos e as emoções, em que o paciente retoma memórias agradáveis, mantém a positividade e consegue expor dúvidas e sentimentos ocultos, mas que denotam grande relevância para o paciente, pois age em situações em que a terapêutica farmacológica não é suficiente, harmonizando suas percepções, o que garante um fortalecimento da relação e maior efetividade para entender as necessidades de cada paciente. (PORTER et al., 2017; PREISSLER et al., 2016; ARCHIE; BRUERA; COHEN, 2013).

Um dos aspectos primordiais considerados na musicoterapia é a instauração da via emocional, a partir das técnicas como a receptiva que auxilia o paciente a retirar o foco da doença e a relaxar com a ajuda da imaginação e a ativa que facilita a expressão não-verbal, esclarecendo muitos medos e angústias a partir da improvisação, por exemplo, ou até mesmo utilizando ambos os recursos somados ou não às interações verbais com o paciente, resultando na liberação das emoções e sentimentos inexplorados que apresentam a capacidade de ampliar o sofrimento emocional e a dor física dos enfermos (RAMIREZ et al., 2018; PORTER et al., 2017; PREISSLER et al., 2016; ARCHIE; BRUERA; COHEN, 2013).

No âmbito fisiológico, há uma possível correlação da música e seus efeitos neurobiológicos, ocorrendo além de uma alteração no sistema autônomo, com a modulação positiva do sistema nervoso parassimpático e negativo do sistema nervoso simpático, a uma melhora de alguns parâmetros como o fluxo sanguíneo e a frequência cardíaca, a música ainda age em todas as estruturas cerebrais límbicas e paralímbicas, especialmente para o corpo estriado ventral, parte do mesencéfalo, a amígdala e o hipocampo, aumentando o fluxo cerebral dos dois primeiros e diminuindo os dos dois últimos. (WARTH et al., 2015; ARCHIE; BRUERA; COHEN, 2013). 
Resultando disso existe uma competição entre a música e os estímulos dolorosos ou nociceptivos o que reduz as taxas de dor e ansiedade, contribuindo para a redução do uso de analgésicos e ansiolíticos pelos pacientes, o que torna plausíveis as investigações com exames de neuroimagem e eletroencefalograma para examinar a correlação entre a música e a alterações em nível de sistema nervoso central (RAMIREZ et al., 2018; WARTH et al., 2015; ARCHIE; BRUERA; COHEN, 2013).

Assim, a música abrange o surgimento de variados sentimentos, que podem modular o estado emocional, proporcionar bem-estar ao paciente, beneficiar na superação dos efeitos emocionais negativos inerentes a sua condição, atuando em várias áreas como o relaxamento, a vitalidade, a diversão, a autorreflexão e a diminuição do estresse. (RAMIREZ et al., 2018; PREISSLER et al., 2016; ARCHIE; BRUERA; COHEN, 2013).

Esse último fator pode ocasionar uma redução do processo inflamatório celular e poderia estar ligado, nos casos de câncer, a uma paralisação do crescimento tumoral e da sua angiogênese, determinando novas rotas que poderiam correlacionar o uso de musicoterapia com redução de mortalidade, porém ainda são necessários estudos adicionais que abordem essa temática (RAMIREZ et al., 2018; PREISSLER et al., 2016; ARCHIE; BRUERA; COHEN, 2013).

Os pacientes que estão em cuidados paliativos, que se propõe a realizar uma identificação das principais dificuldades que o enfermo enfrenta, muitas vezes, anseiam em manter uma boa qualidade de vida, assumindo dessa forma o controle da sua existência deles, encontram dificuldade em tê-la, pois, o uso de muitas medicações para o controle da dor, levam a muitos efeitos colaterais que reduzem essa qualidade de vida almejada. Assim, a musicoterapia, em conjunto com os cuidados paliativos, possui estratégias que facilitam esse manejo dos sintomas álgicos, principalmente quando o terapeuta consegue absorver a identidade musical já própria do paciente, criando o forma de aplicação da terapia e as metas em conjunto com o paciente, levando em consideração o contexto sociocultural desse paciente e tendo um maior impacto com o uso da música (SILVA; ALVIN; MARCON, 2014; SILVA; SALES, 2013; GUTGSELL et al., 2013).

Considerando o exposto acima, os cuidados paliativos associados à musicoterapia fornecem, além de uma visão mais humanizada e abrangente do paciente e da sua família, uma alternativa complementar, sendo a segunda forma mais comum dentre as práticas utilizadas nos cuidados paliativos. Os benefícios desta prática são reconhecidos tanto pela equipe de saúde dos cuidados paliativos como pela maioria dos pacientes e é diferenciada das outras técnicas 
musicais pela presença de um musicoterapeuta que adapta suas metas de acordo com as peculiaridades dos clientes, objetivando resultados específicos. (PORTER et al., 2017; MCCONNEL et al., 2016; WARTH et al., 2015). Dessa forma, para elucidar as principais questões dos cuidados paliativos, segue a Figura 4.

Figura 4 - Mapa conceitual sobre cuidados paliativos

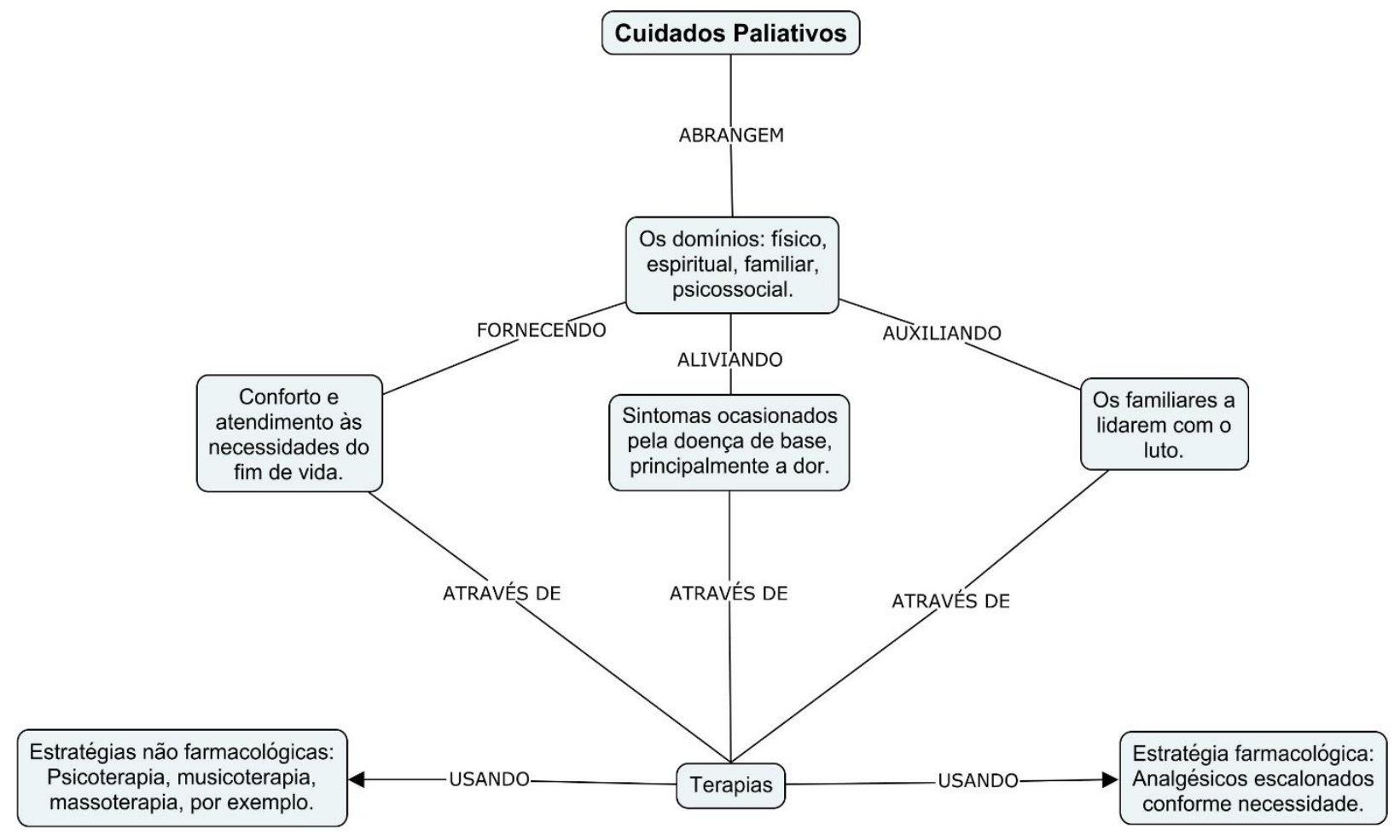

Fonte: Autoria própria

\section{Conclusões}

É importante mencionar que as atuações da musicoterapia percorrem as trajetórias de manejo da dor e do sofrimento psíquico, favorecendo o suporte clínico às situações limítrofes de final de vida. A aplicabilidade suscita o aprimoramento técnico e operacional dos profissionais envolvidos, em particular, no mapeamento de sinais e controle da "terminalidade" em si, e o do enquadre situacional da doença. 
Neste contexto, a música age fomentando a comunicação e conexão interpessoal, o que facilita a abordagem a assuntos de natureza física, psíquica, espiritual e social por meio de terapias que utilizam variadas técnicas ativas e receptivas.

Considerando que apenas o estudo de Warth, realizado em 2015, não apresentou resultados positivos quanto ao uso da musicoterapia em detrimento de outras técnicas de relaxamento, vale ressaltar que o número de pacientes utilizados eram apenas 9 e que as sessões não foram concluídas com todos os participantes, a musicoterapia emerge como uma terapia não farmacológica plausível para os cuidados paliativos na terminalidade da vida.

\section{Referências}

ARCHIE P.; BRUERA E.; COHEN L. Music-based interventions in palliative cancer care: a review of quantitative studies and neurobiological literature. Support Care Cancer. 2013;21(9):2609-24.

BRADT, Joke; DILEO, Cheryl. Music therapy for end-of-life care. Cochrane Database Of Systematic Reviews, [s.l.], p.1-36, 20 jan. 2010. John Wiley \& Sons, Ltd.

BUNN, Frances et al. Improving living and dying for people with advanced dementia living in care homes: a realist review of Namaste Care and other multisensory interventions. Bmc Geriatrics, [s.1.], v. 18, n. 1, p.1-15, dez. 2018. Springer Science and Business Media LLC.

CAIRES, Juliana Souza et al. A UTILIZAÇÃO DAS TERAPIAS COMPLEMENTARES NOS CUIDADOS PALIATIVOS: BENEFÍCIOS E FINALIDADES*. Cogitare Enferm, [s.1.], v. 3, n. 19, p.514-520, set. 2014.

DE-LA-TORRE-UGARTE-GUANILO, M. C.; TAKAHASHI, R. F.; BERTOLOZZI, M. R. Revisão sistemática: noções gerais. Revista da Escola de Enfermagem USP, São Paulo, v. 45, n. 5, p. 1260 - 1266, out. 2011.

DIETRICH, Cordula et al. Treating palliative care patients with pain with the body tambura: A prospective case study at St. Joseph's hospice for dying destitute in Dindigul South India. Indian Journal Of Palliative Care, [s.1.], v. 21, n. 2, p.236-241, maio/ago. 2015. Medknow.

FERNANDO, Gunasekara Vidana Mestrige Chamath et al. "Adjunctive Effects of a Short Session of Music on Pain, Low-mood and Anxiety Modulation among Cancer Patients" - A Randomized Crossover Clinical Trial. Indian Journal Of Palliative Care. [s.1.], p. 367-373. set. 2019. Acesso em: 28. Dez. 2019

GUTGSELL, Kathy Jo et al. Music Therapy Reduces Pain in Palliative Care Patients: A Randomized Controlled Trial. Journal Of Pain And Symptom Management, [s.1.], v. 45, n. 5, p.822-831, maio 2013. Elsevier BV.

GIL, Antônio Carlos. Como Encaminhar uma Pesquisa. In: GIL, Antônio Carlos. Como elaborar projetos de pesquisa. São Paulo: Atlas S.a., 2017. p. 17-21. 
KAURA, Amit. Revisão Sistemática e Metanálise. In: KAURA, Amit. Medicina Baseada em Evidências: Leitura e Redação de Texto Clínico. Rio de Janeiro: Elsevier, 2016. Cap. 4. p. 4354.

KORCZAK D.; WASTIAN M.; SCHNEIDER M. Music therapy in palliative setting. GMS Health Technol Assess. 2013;9:Doc07. Disponível em: https://www.ncbi.nlm.nih.gov/pmc/articles/PMC3728641/. Acesso em: 27. Dez. 2019.

KRISHNASWAMY P.; NAIR S. Effect of music therapy on pain and anxiety levels of cancer patients: A pilot study. Indian J Palliat Care. 2016; 22:307-11. Disponível em: https://www.ncbi.nlm.nih.gov/pubmed/27559260. Acesso em: 27. Dez. 2019.

MCCONNELL, Tracey et al. Evaluation of the Effectiveness of Music Therapy in Improving the Quality of Life of Palliative Care Patients: A Randomised Controlled Pilot and Feasibility Study. Pilot and Feasibility Studies 2. 2016: 70. PMC. Disponível em: https://www.ncbi.nlm.nih.gov/pubmed/27965885. Acesso em: 27. Dez. 2019..

PORTER, Sam et al. A critical realist evaluation of a music therapy intervention in palliative care. Bmc Palliative Care, [s.1.], v. 16, n. 1, p.1-12, dez. 2017. Springer Science and Business Media LLC.

PORTER, Sam et al. A randomised controlled pilot and feasibility study of music therapy for improving the quality of life of hospice inpatients. Bmc Palliative Care, [s.1.], v. 17, n. 1, p.1-9, 27 nov. 2018. Springer Science and Business Media LLC.

PREISSLER, Pia et al. Favored subjects and psychosocial needs in music therapy in terminally ill cancer patients: a content analysis. BMC palliative care, 15, 48. 2016. Disponível em: https://bmcpalliatcare.biomedcentral.com/articles/10.1186/s12904-016-0122-7. Acesso em: 27. Dez. 2019.

RAMIREZ, Rafael et al. EEG-Based Analysis of the Emotional Effect of Music Therapy on Palliative Care Cancer Patients. Frontiers in Psychology. 9:254. 2018. Disponível em: https://www.ncbi.nlm.nih.gov/pmc/articles/PMC5840261/. Acesso em: 27. Dez. 2019.

SAMPAIO, R. F.; MANCINI, M. C. Estudos de Revisão Sistemática: um guia para síntese criteriosa da evidência científica. V. 11, n. 1. São Carlos-SP: Revista Brasileira de Fisioterapia, p. 83-89, 2007.

SEKI, N.H.; GALHEIGO, S.M. The use of music in palliative care: humanizing care and facilitating the farewell. Interface - Comunic., Saude, Educ., v.14, n.33, p.273-84, abr./jun. 2010.

SILVA, Vladimir Araujo da; SALES, Catarina Aparecida. Encontros musicais como recurso em cuidados paliativos oncológicos a usuários de casas de apoio. Revista da Escola de Enfermagem da Usp, [s.1.], v. 47, n. 3, p.626-633, jun. 2013. FapUNIFESP (SciELO). http://dx.doi.org/10.1590/s0080-623420130000300015. Acesso em: 28. Dez. 2019

SILVA, Vladimir Araujo de; ALVIM, Neide Aparecida Titonelli; MARCON, Sonia Silva. Significados e sentidos da identidade musical de pacientes e familiares sob cuidados paliativos oncológicos. Revista Eletrônica de Enfermagem, [s.1.], v. 16, n. 1, p.1-10, 31 mar. 2014. Universidade Federal de Goias. 
SCHMID, W. et al. Patient's and health care provider's perspectives on music therapy in palliative care - an integrative review. BMC Palliative Care. 2018. Disponível em: https://www.ncbi.nlm.nih.gov/pmc/articles/PMC5819707/. Acesso em: 27. Dez. 2019.

SCHMID, Wolfgang; OSTERMANN, Thomas. Home-based music therapy - a systematic overview of settings and conditions for an innovative service in healthcare. Bmc Health Services Research, [s.1.], v. 10, n. 1, p.1-10, 14 out. 2010. Springer Science and Business Media LLC.

TEUT, Michael et al. Perceived outcomes of music therapy with body tambura in end of life care a qualitative pilot study. BMC Palliat Care. 2014;13(1):18.

WARTH, Marco et al. "Song of Life (SOL)" study protocol: a multicenter, randomized trial on the emotional, spiritual, and psychobiological effects of music therapy in palliative care. Bmc Palliative Care, [s.1.], v. 18, n. 1, p.1-11, 30 jan. 2019. Springer Science and Business Media LLC.

WARTH, Marco et al. Trajectories of Terminally Ill Patients' Cardiovascular Response to Receptive Music Therapy in Palliative Care. Journal Of Pain And Symptom Management, [s.1.], v. 52, n. 2, p.196-204, ago. 2016. Elsevier BV. http://dx.doi.org/10.1016/j.jpainsymman.2016.01.008. Acesso em: 28. Dez. 2019.

WARTH, Marco et al. Music therapy to promote psychological and physiological relaxation in palliative care patients: protocol of a randomized controlled trial. BMC Palliative Care. 2014;13:60. Disponível em: https://bmcpalliatcare.biomedcentral.com/articles/10.1186/1472684X-13-60 .Acesso em: 27. Dez. 2019.

WARTH, Marco et al. Music therapy in palliative care - a randomized controlled trial to evaluate effects on relaxation. Dtsch Arztebl Int 2015; 112: 788-94.

WARTH, Marco et al. Effects of vibroacoustic stimulation in music therapy for palliative care patients: a feasibility study. BMC Complementary and Alternative Medicine. 2015;15:436. Disponível em: https://www.ncbi.nlm.nih.gov/pmc/articles/PMC4681146/. Acesso em: 27. Dez. 2019.

World Health Organization. Palliative Care. 2018. Disponível em: <http://www.who.int/en/newsroom/fact-sheets/detail/palliative-care>. Acesso em: 27. Dez. 2019.

ZENG, Yvette S. et al. Complementary and Alternative Medicine in Hospice and Palliative Care: A Systematic Review. Journal Of Pain And Symptom Management, [s.1.], v. 56, n. 5, p.781-794, nov. 2018. Elsevier BV.

\section{Como citar este artigo (Formato ABNT):}

AGUILERA, Kevellyn Cruz; MENDES, Isabelle Lima; ROLIM NETO, Modesto Leite. O uso da Música nos Cuidados Paliativos. Id on Line Rev.Mult. Psic., Fevereiro/2020, vol.14, n.49, p. 229-247. ISSN: 1981-1179.

Recebido: 22/01/2020;

Aceito: 28/01/2020. 